\title{
Roux-en-Y gastric bypass in a patient with situs inversus totalis - a case report
}

\author{
Piotr Kalinowski, Rafał Paluszkiewicz, Piotr Remiszewski, Mariusz Grodzicki, Bartosz Cieślak, Krzysztof Dudek, \\ Marek Krawczyk \\ Department of General, Transplant and Liver Surgery, Medical University of Warsaw, Poland
}

Videosurgery and other miniinvasive techniques 2010; 5 (3): 110-114 DOI: 10.5114/wiitm.2010.16423

\begin{abstract}
We report a case of a Roux-en-Y gastric bypass (RYGB) in a morbidly obese patient with situs inversus totalis. Current experience with bariatric surgery in patients with situs inversus totalis shows that they can be operated on safely. The open approach in this case of a difficult anatomy may be beneficial and safer for the patient. The technique itself requires only minor modifications. The results of bariatric surgery in terms of weight loss are comparable to the general population of morbidly obese patients after RYGB.
\end{abstract}

Key words: Roux-en-Y gastric bypass, RYGB, open gastric bypass, situs inversus, bariatric surgery

\section{Introduction}

Situs inversus is a congenital anomaly characterized by a mirror image orientation of inner organs in gross anatomical appearance. However, there is also a cluster of underlying genetic abnormalities leading to several disorders related primarily to defects in ciliary function [1]. The incidence of situs inversus totalis (complete transposition of all the viscera) is estimated to be $0.01 \%$ of the general population. Situs inversus shows high correlation with complex congenital heart abnormalities, bilateral superior vena cava, splenic agenesis or polysplenia, absence of inferior vena cava, symmetrical or midline liver, intestinal malrotation and other less frequently encountered abnormalities [2, 3]. Partial visceral transposition most often involves the heart. Dextrogastria as an isolated abnormality may also be encountered although less frequently. The presence of situs inversus represents a challenge to a surgeon, especially when operating on asymmetrical structures or single unpaired organs that may be altered. The anomaly is not a contraindication to surgical treatment and various surgical procedures have been performed in patients with situs inversus ranging from laparoscopic cholecystectomy $[4,5]$ to the most complicated ones such as oesophagectomy [6] or liver transplantation [7, 8]. Nonetheless, surgical experience in patients with situs inversus is limited, and only a few cases have been reported to date on bariatric surgery in such patients. In Poland the only published cases of stomach surgery in patients with situs inversus were related to gastric cancer [9]. Today many surgical centres in Poland offer bariatric surgery to their patients and report their results [10-13] but to our best knowledge the presented case is the first case of bariatric surgery in a patient with situs inversus in Poland.

\section{Case report}

A 27-year-old female was referred to the Department of General, Transplant and Liver Surgery, Medical University of Warsaw for weight loss surgery. Her 
BMI was $59.1(155 \mathrm{~kg} / 162 \mathrm{~cm})$ and she had several concomitant disorders including hypertension, history of chronic upper respiratory tract infections, bilateral hearing impairment, primary ciliary dyskinesia (PCD) and situs inversus totalis. She had already made several attempts at weight loss using low calorie diets, commercially available dieting programmes and drugs such as orlistat and sibutramine. She had no previous abdominal surgery. After a thorough preoperative workup adopted at our institution, that was described before [14], she was qualified as a candidate for gastric bypass surgery. Preoperative imaging studies showed dextrocardia on chest $x$-ray (Figure 1) and a mirror orientation of abdominal organs on abdominal ultrasound. In order to assess other possible abnormalities computed tomography (CT) of the thorax and abdomen was performed (Figures 2, 3). Upper gastrointestinal endoscopy showed no abnormalities. She had no history of bowel obstruction that might suggest bowel malrotation. The results of spirometry were at the lower limit of normal. The three bariatric operations most commonly performed today [15] are available in our department: Roux-en-Y gastric bypass, laparoscopic sleeve gastrectomy and laparoscopic adjustable gastric banding. However, the open gastric bypass technique was chosen in order to achieve maximum safety and have better control of possible intraoperative

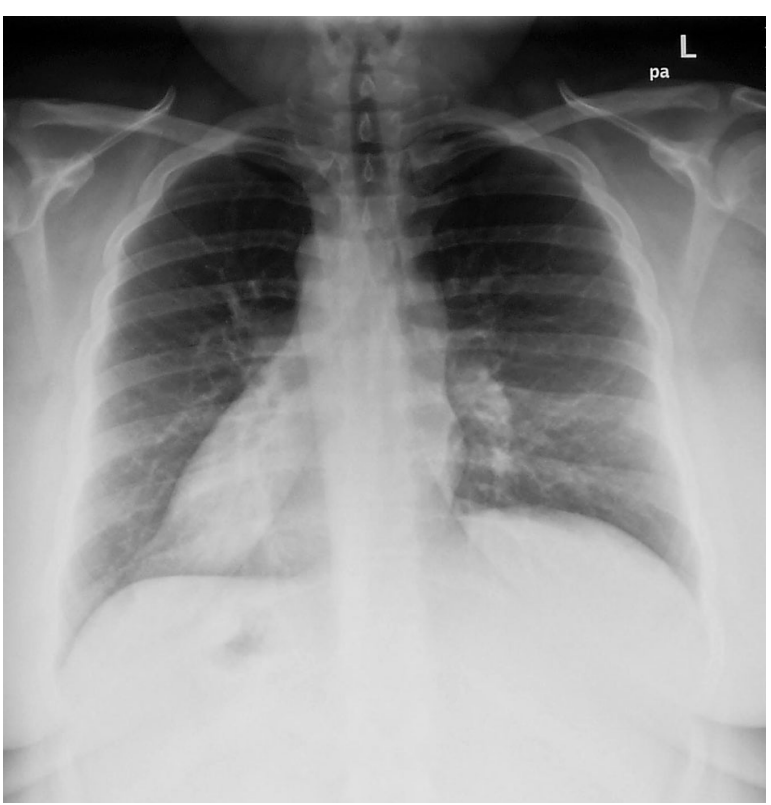

Figure 1 . The chest $x$-ray of the patient shows dextrocardia complications related to abnormal anatomy. The patient was evaluated by an anaesthesiologist the day before surgery. The surgery was performed under combined general and epidural anaesthesia. Open Roux-en-Y gastric bypass was performed with the standard technique adopted at our department:

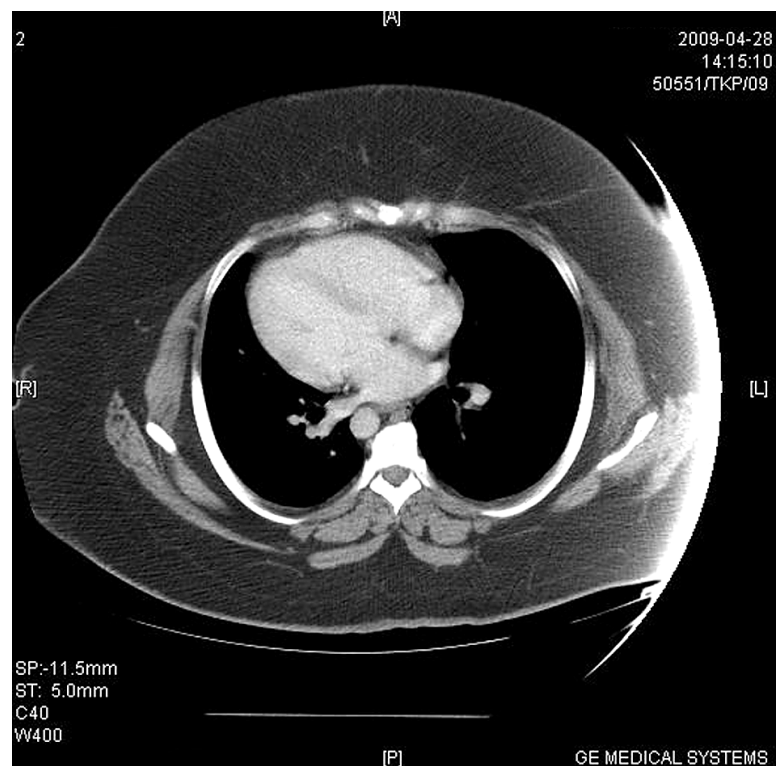

Figure 2. CT scan of the chest confirms dextrocardia. There are no other abnormalities detected in the thoracic organs

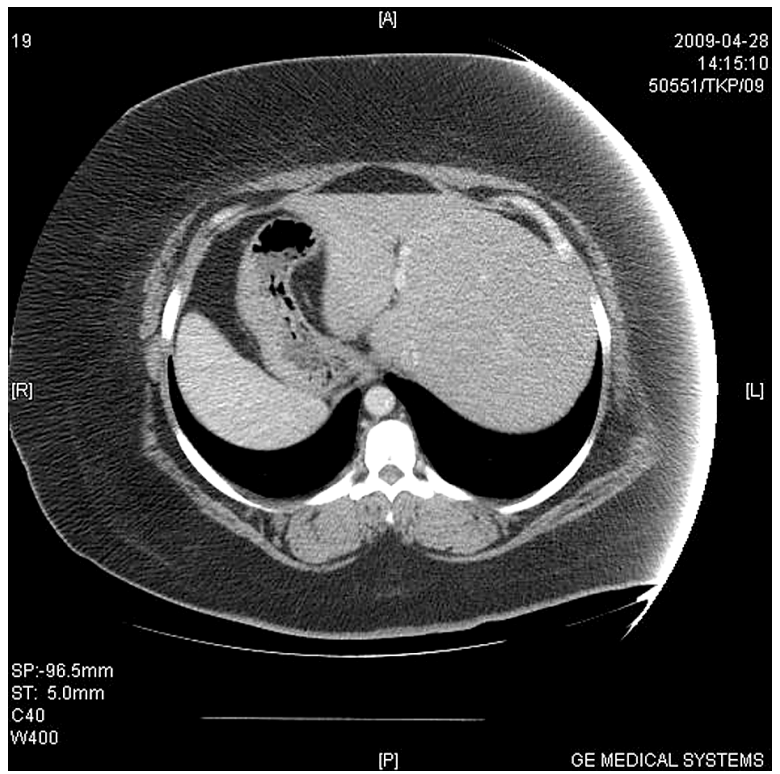

Figure 3. CT scan of the abdomen shows mirror position of the liver, the stomach and the spleen characteristic of situs inversus totalis 


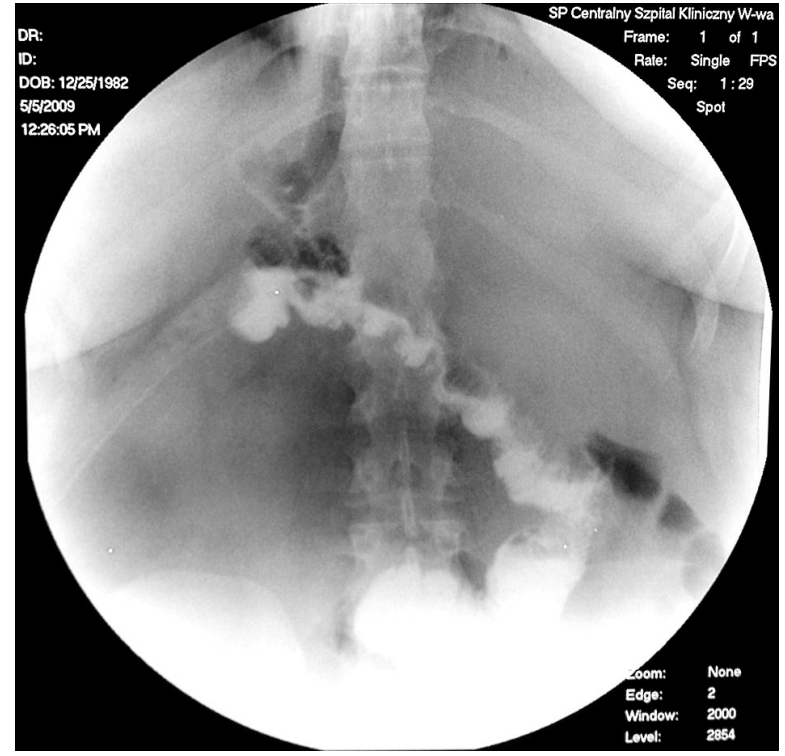

Figure 4. Postoperative upper GI study with water-soluble contrast. There is no problem with the gastrointestinal anastomosis but the limb of the jejunum follows a mirrored course from the upper right to the lower left

laparotomy through bilateral subcostal incision, antecolic and antegastric placement of the Roux limb and hand-sewn gastrointestinal anastomosis. In this case there was no need to mobilize the smaller lobe (in regular anatomy the left lobe) of the liver to achieve better exposure of the stomach. Sometimes this technique is helpful in cases of large, steatotic livers with the left lobe hindering access to the fundus of the stomach. In situs inversus the anatomy of the liver may be abnormal with a large "smaller" lobe or sometimes even symmetrical midline liver, and freeing the smaller lobe from its triangular ligament may be an essential step in this operation. The stomach showed no abnormality except the complete mirror position. Palpation of the hepato-gastric ligament did not reveal any abnormal or accessory blood vessels. We used a $90 \mathrm{~mm}$ green load stapler (TA90B, Tyco) to transect the stomach just below the cardia, creating a 15-20 ml pouch. The small bowel was divided $100 \mathrm{~cm}$ from the ligament of Treitz and a $100 \mathrm{~cm}$ Roux limb was created. The Roux limb was placed in the antecolic and antegastric position. Both jejunojejunal and gastro-jejunal anastomoses were handsewn. In the operating room there were only minor modifications in the setup. The surgeon was on the right side of the patient and both assistants were positioned on the left, which is no different than the normal setting. The smaller lobe of the liver (corresponding to the left lobe in a typical anatomy) extending to the right side had to be retracted from the left, but we did not have to use any special instruments. The whole procedure was performed in a mirror-image manner. The postoperative course was uneventful and the patient was discharged on the fifth postoperative day after a satisfactory result of an upper GI series with a water-soluble contrast medium (Figure 4). During the follow-up the patient's BMI decreased from 59.1 at baseline to 47 at 3 months. After 6 months her BMI was 41 and after 12 months it was 39.

\section{Discussion}

There was only one patient with situs inversus in the group of 181 bariatric patients operated on in our department (0.55\%). That kind of anatomical anomaly has not been shown to be associated with morbid obesity so the expected incidence is equal to that of the general population (0.01\%). Patients with situs inversus should be evaluated thoroughly for any potentially serious respiratory or cardiac abnormalities. In cases of situs inversus totalis the diagnosis is usually made early during the initial evaluation. However, there have also been reports of rare cases of isolated abnormal position of the stomach that could make bariatric procedures extremely difficult [16]. These included incomplete rotation of the stomach with an abnormal position behind or above the liver, sometimes even with diaphragmatic hernia and displacement of the stomach into the thorax. Malrotation of the small intestine with abnormalities in the intestinal vasculature may also make surgery impossible. During the initial workup of the patient it is important to assess pulmonary function with spirometry because of a high risk of pulmonary complications related to impaired function of the epithelium of the respiratory tract. The endoscopist has to pay special attention during upper Gl endoscopy and look for hiatal hernias and an abnormal appearance of the stomach. In our department we observe the rule that a surgeon performs an upper Gl endoscopy. In order to assess possible anatomical variations and unexpected pathology it is necessary to obtain a CT scan of the thorax and abdomen. There is no evidence that situs inversus itself creates an increased risk for surgical complications and documented cases of 
bariatric surgery, although scarce, did not show any complications specific to this anomaly. That kind of patient may be a challenge also for an anaesthesiologist because of concomitant respiratory problems. Pauli et al. [18] reported a case of a super-obese patient with severe pulmonary disease that needed weight loss in order to qualify for lung transplantation. Surgeons have to pay attention to abnormal anatomy and in the case of gastric bypass surgery especially to stomach or intestine malrotation, polysplenia or atypical liver anatomy and blood supply (i.e. the accessory hepatic artery branching from the left gastric artery). In the published literature, there have been only 5 reports of laparoscopic adjustable gastric banding [17-21], 1 report of laparoscopic sleeve gastrectomy [22] and 2 reports of laparoscopic Rouxen-Y gastric bypass $[23,24]$ in patients with situs inversus. Atypical organ orientation may be a problem for a laparoscopic surgeon and in that case a minimally invasive procedure requires more technical and mental preparation of the surgeon and staff of the operating theatre. Ahmed and O'Malley [24] underline the need for a mirror approach during laparoscopy, which can be really challenging with an already complex laparoscopic procedure. The authors find this technique feasible but report 1.5 times longer operative time (160 min vs. 105 min) than in a patient with typical anatomy, which may reflect possible difficulties encountered during surgery. This is still a better result than with an open approach, which takes longer (in our series of open gastric bypass the average time was 190 min and in this case of situs inversus it was 240 min). However, the open approach does not require much modification and the position of the surgeon does not have to be changed. It enables better inspection of the operating field with the possibility of palpation, which is important because of possible abnormalities in vasculature. The final position of the small gastric pouch and the jejunum is mirrored but in fact surgical manoeuvres do not require switching hands, using any special instruments or radically changing the operating room setup. The difficulty of using the right hand in place of the left hand during laparoscopic sleeve gastrectomy in a patient with situs inversus was mentioned by Catheline et al. [22]. However, an obvious benefit of laparoscopy is that a procedure can be converted into open surgery. We believe that the safety of the patient is crucial and an elective open approach may be beneficial for the patient and more comfortable for the surgeon.

\section{References}

1. Bush A, Chodhari R, Collins N, et al. Primary ciliary dyskinesia: current state of the art. Arch Dis Child 2007; 92: 1136-40.

2. Campbell M, Deuchar DC. Absent inferior vena cava, symmetrical liver, splenic agenesis, and situs inversus, and their embryology. Brit Heart J 1967; 29: 268.

3. Chandraraj S. Observations on some additional abnormalities in Situs inversus viscerum. J Anat 1976; 122: 377-88.

4. Kumar S, Fusai G. Laparoscopic cholecystectomy in situs inversus totalis with left-sided gall bladder. Ann R Coll Surg Engl 2007; 89: W16-8.

5. Mitura K, Romańczuk M, Blicharz P, Boruciński M. Laparoscopic cholecystectomy in patient with situs viscerum inversus. Videosurgery and other miniinvasive techniques 2006; 1: 40-2.

6. Mimae T, Nozaki I, Kurita A, et al. Esophagectomy via left thoracotomy for esophageal cancer with situs inversus totalis: report of a case. Surg Today 2008; 38: 1044-7.

7. Wei JM, Liu YN, Qiao JC, et al. Liver transplantation in a patient with situs inversus: a case report. Chin Med I 2007; 120: 1376-7.

8. Klintmalm GB, Bell MS, Husberg BS, et al. Liver transplant in complete situs inversus: a case report. Surgery 1993; 114: 102-6.

9. Bielecki K, Gregorczyk M, Baczuk L. Visceral situs inversus in three patients. Wiad Lek 2006; 59: 707-9.

10. Kwiatkowski A, Paśnik K, Stanowski E, Badyda A. Regression of metabolic syndrome depending on type of bariatric surgery. Videosurgery and other miniinvasive techniques 2009; 4: 53-8.

11. Hady RH, Dadan J, Iwacewicz P. Evaluation of the efficiency of chosen methods of surgical treatment of pathological obesity in our own material. Videosurgery and other miniinvasive techniques 2008; 3: 39-44.

12. Szydłowski K, Orłowski M, Frask A, et al. Complications after surgical treatment of obesity based on own material. Videosurgery and other miniinvasive techniques 2008; 3: 45-52.

13. Szewczyk T, Janczak P, Duszewski M, Modzelewski B. Technical problems in laparoscopic sleeve gastrectomy. Videosurgery and other miniinvasive techniques 2009; 4: 95-101.

14. Paluszkiewicz R, Kalinowski P, Remiszewski P, et al. Early results of Roux-en-Y gastric bypass in patients with morbid obesity. Videosurgery and other miniinvasive techniques 2006; 1: 150-7.

15. Dadan J, Iwacewicz P, Hady RH. New approaches in bariatric surgery. Videosurgery and other miniinvasive techniques 2008; 3: 66-70.

16. Teplick JG, Wallner RJ, Levine AH, et al. Isolated dextrogastria: report of two cases. Am J Roentgenol 1979; 132: 124-6.

17. Ersoy E, Koksal H, Ege B. Laparoscopic gastric banding for morbid obesity in a patient with situs inversus totalis. Obes Surg 2005; 15: 1344-6.

18. Pauli EM, Wadiwala II, Rogers AM. Laparoscopic placement of an adjustable gastric band in a super-super obese patient with situs inversus. Surg Obes Relat Dis 2008; 4: 768-9.

19. Samaan M, Ratnasingham A, Pittathankal A, et al. Laparoscopic adjustable gastric banding for morbid obesity in a patient with situs inversus totalis. Obes Surg 2008; 18: 898-901.

20. Matar ZS. Laparoscopic adjustable gastric banding in a morbidly obese patient with situs inversus totalis. Obes Surg 2008; 18: $1632-5$. 
21. Taskin M, Zengin K, Ozben V. Concomitant laparoscopic adjustable gastric banding and laparoscopic cholecystectomy in a super-obese patient with situs inversus totalis who previously underwent intragastric balloon placement. Obes Surg 2009; 19: 1724-6.

22. Catheline JM, Rosales C, Cohen R, et al. Laparoscopic sleeve gastrectomy for a super-super-obese patient with situs inversus totalis. Obes Surg 2006; 16: 1092-5.

23. Wittgrove AC, Clark GW. Laparoscopic gastric bypass for morbid obesity in a patient with situs inversus. J Laparoendosc Adv Surg Tech A 1998; 8: 53-5.

24. Ahmed AR, O'Malley W. Laparoscopic Roux-en-Y gastric bypass in a patient with situs inversus. Obes Surg 2006; 16: 1392-4. 